\title{
Front Environment Recognition of Personal Vehicle using the Image Sensor and Acceleration Sensors for Everyday Computing
}

\author{
Takahiro Matsui ${ }^{1}$, Takeshi Imanaka ${ }^{1}$, Yasuyuki Kono ${ }^{1}$, \\ ${ }^{1}$ Graduate School of Science and Technology, Kwansei Gakuin University, 2-1 \\ Gakuen, Sanda, Hyogo, JAPAN \\ $\{$ acl27598, bbv84420, kono\}@kwansei.ac.jp
}

\begin{abstract}
In this research, we propose the method for detecting moving objects in front of the Segway by detecting running state for the Segway. Running state of the personal vehicle Segway is detected with both an image sensor and an acceleration sensor mounted on the Segway. When objects are moving in front of the Segway, the image sensor can capture the motion while the acceleration sensor shows a different result. By analyzing the difference our method successfully recognizes moving objects from environment.
\end{abstract}

Keywords: Segway, Image Sensor, Acceleration Sensor, Optical Flow.

\section{Introduction}

This research examines the safety and comfort of the personal vehicle Segway [1] which uses electrical energy instead of gasoline in everyday environment. Unlike other vehicles such as cars and bicycle, the Segway can be used to run not only on the road in outdoors, but also indoors such as inside the buildings or factories shown in Fig. 1 right. Safety must be especially considered for the Segway when driving in such place, since they have high pedestrian traffic. To improve the safety of the Segway, a system to notify the danger to the rider and avoid the incident automatically is necessary. This paper proposes the method to detect moving objects in front of the Segway. Rider can easily recognize static obstacles and avoid them, but it is difficult to avoid dynamic obstacles such as unexpected pedestrian and other personal vehicles. Thus, if a system can recognize these obstacles and notify the riders or avoid them automatically, safety of the Segway can be assured. Most of the researches which use vehicle video system to recognize the front environments are instead for use of cars, and are not applicable for slow vehicle like the Segway, which requires to recognize dynamic obstacles in close range. Moreover, the operation of the Segway is different from conventional vehicles in the way that has no acceleration pedal and no brakes. A rider stands on the footplate between two wheels to adjust speed, movement, and direction of the Segway. Vehicles other than the Segway do not have these features. 
Our proposed method detects the running state of Segway by employing both an image sensor(camera) aimed forward and an acceleration sensor. By integrating sensor readings, our method detects moving objects in the frontal direction. In this research, we focus on the tilting of the handle bar while driving the Segway. From the image sensor mounted on the handle bar, the system analyzes the video captured from it and detects the optical flow [2,3] to obtain the running state among the following six states: forward, backwards, right turn, left turn, acceleration and deceleration. When a moving object such as a walking person is within the range of the image sensor in front of the Segway, it affects the trend of the optical flow, although the readings from acceleration sensors are not affected by the object. Using these data obtained from both sensors, we analyze whether the moving object actually exists within the certain range which the incompatible information has been detected. This method will contribute for safety in the future, notifying the riders to avoid the moving obstacles in front of the Segway.

Place where persons do not exist together to car

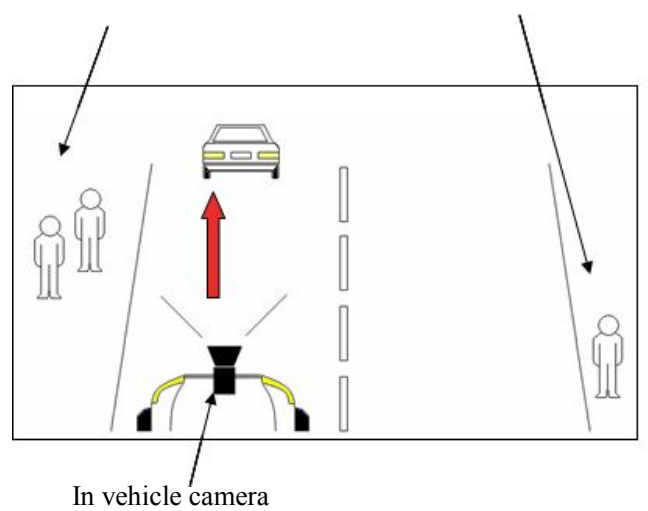

Place where person exists together to vehicle

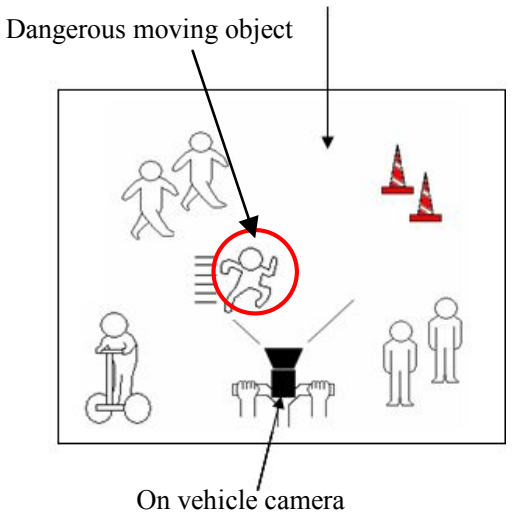

Fig. 1. A difference between a car and the Segway

\section{Research Background}

\subsection{Related Works}

Many of related works usually mount the image sensor on the car. Although the posture of the camera is overall stable in these works, that on the Segway changes depending on the running state, i.e., the rider's center of gravity. For example, when the rider wants to turn right, he tilts the handle bar to the right, and the image sensor also tilts to the right. The rider leans forward to gain speed, so the handle bar together with the camera also leans forward. It is necessary to analyze the running state of Segway by detecting the tilt of the image sensor which is affected by the change of the Segway posture. 
A method for detecting three-dimensional position of moving objects with an invehicle camera is proposed [4]. The method in [4] is not suitable to the Segway because the posture of the camera is not stable while driving. Another approach is to detect approaching vehicles by classifying horizontal edges in an image into two classes; ones on the ground and one above the ground [5]. The method in [5] is also difficult to apply to complicated everyday scenes.

\subsection{Preparation}

The image sensor is mounted on the handle bar as indicated in Fig. 2(A). The acceleration sensor is mounted on the footplate. The image sensor is Glasshopper of Point Grey Research Inc shown in Fig. 3. The acceleration sensor is WAA-001 of Wireless Technologies Inc shown in Fig. 4. The specification of the sensors is shown in Table 1 and 2 [6] [7].

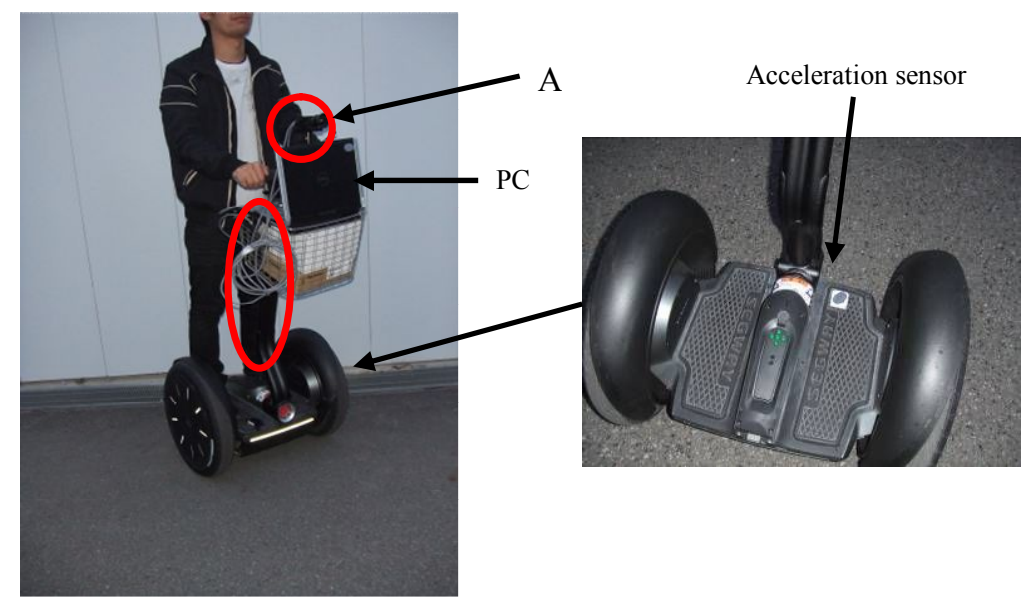

Fig. 2. Placement of sensors on the Segway

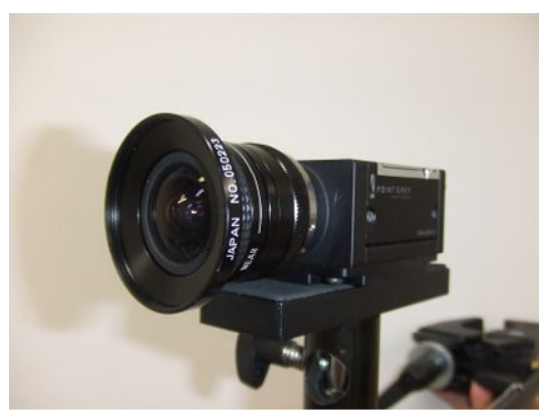

Fig. 3. Grasshopper

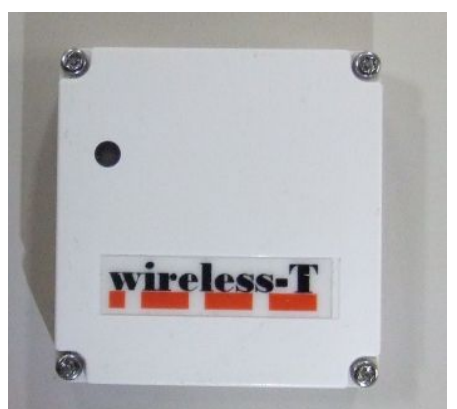

Fig. 4. WAA-001 
Table 1. Spec of the Grasshopper

\begin{tabular}{ll}
\hline & GRAS-20S4C \\
\hline Sensor & Sony 1/1.8 progressive scan CCDs \\
Sampling rate & $30 \mathrm{FPS}$ \\
Resolution & $1624 \times 1224$ \\
Interface & Dual 9 -pin IEEE-1394b $800 \mathrm{Mb} / \mathrm{s}$ ports \\
Dimensions & $44 \times 29 \times 58 \mathrm{~mm}$ \\
Weight & $104 \mathrm{~g}$ (The optical system is excluded) \\
\hline
\end{tabular}

Table 2. Spec of the WAA-001

\begin{tabular}{ll}
\hline \multicolumn{2}{c}{ WAA-001 } \\
\hline Sampling rate & MAX 200Hz \\
Range of detection & $\pm 3 \mathrm{~g}$ \\
Interface & Bluetooth (Class 2) \\
Dimensions & $38 \times 39 \times 10 \mathrm{~mm}$ \\
Weight & $17 \mathrm{~g}$ \\
\hline
\end{tabular}

\section{Moving Object Detection by Sensor Fusion}

In this research, the image sensor is mounted on the handle bar of the Segway and the acceleration sensor is mounted on the footplate between two wheels. Running states of the Segway are detected from these sensors, and moving objects are recognized from the discrepancy of the detection results.

The image sensor detects and analyzes optical flow patterns of each running states while the acceleration sensor measures the acceleration of the Segway to determine the running states. The optical flow shows the movement of feature points between video frames expressed as vector. Considering the running state of the Segway as moving forward, when the image sensor detects no moving objects, the optical flow displays as shown in Fig. 5 left. When a moving object is within the field of view, the optical flow is disrupted as shown in Fig. 5 right. On the other hand, the moving object has no influence on the detection results of the acceleration sensor, which measures the acceleration of the Segway for detection of the running states.
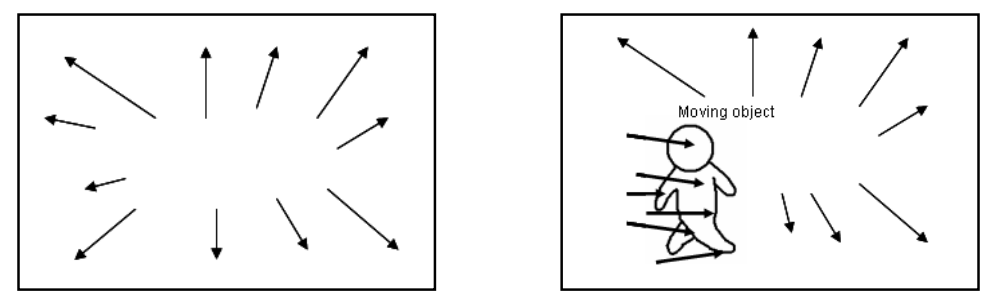

Fig. 5. Optical flows change when a moving object is recognized 


\section{Running state Detection using each Sensor}

\subsection{Posture Change and Running State of the Segway}

The method to detect the running state is described here, namely; forwards, backwards, right turn, left turn, acceleration and deceleration.

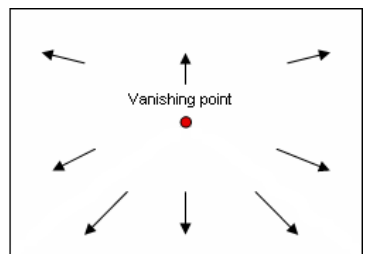

Fig. 6. Forward

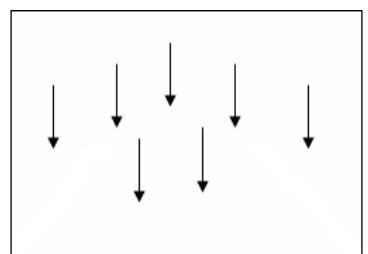

Fig. 9. Deceleration

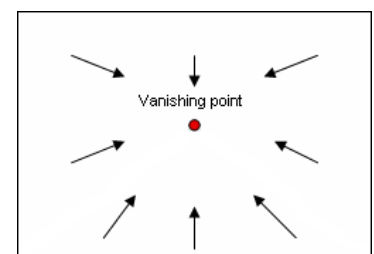

Fig. 7. Backwards

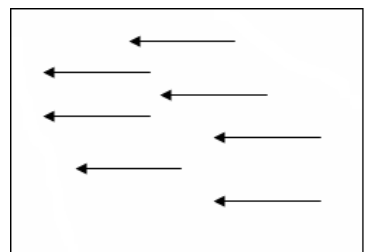

Fig. 10. Right turn

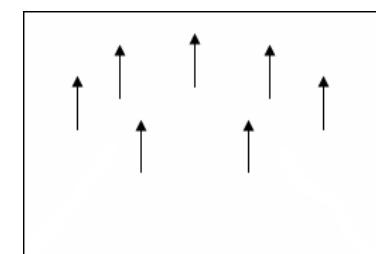

Fig. 8. Acceleration

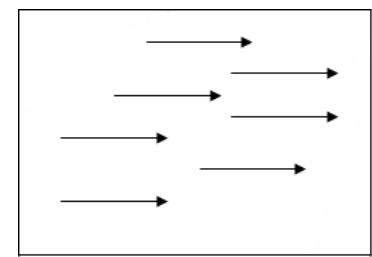

Fig. 11. Left turn

Details of optical flow pattern for each running state is described as follows.

In forward running state, a vanishing point and optical flows appear as shown in Fig. 6. In backwards running state, a vanishing point and optical flows appear as shown in Fig. 7. In accelerating running state, optical flows appear as shown in Fig. 8. The flows rise from the bottom to the top as the handle bar leans forward. The camera is faced downwards and a range of the image sensor moves to bottom. In deceleration running state, optical flows appear as shown in Fig. 9. The flows descend from top to bottom as the handle bar is pulled back, and the camera is faced upward. In addition, after the Segway has decelerated, note that the posture of the Segway returns to the upright position. In turning right running state, optical flows appear as shown in Fig. 10. In turning left running state, optical flows appear as shown in Fig. 11.

\subsection{Running States Detection using the Acceleration Sensors}

When running state of the Segway changes, the acceleration sensor data change characteristically. Fig. 13 shows one example of the acceleration data. In addition, the line $\mathrm{X}$ shows the acceleration that is traveling direction of the Segway, and the line $\mathrm{Y}$ shows the acceleration that is horizontal direction and vertical to $\mathrm{X}$ axis against 
traveling direction of the Segway shown in Fig. 12. The acceleration data change remarkably when the running state changes.

For example, when the vehicle is running forward, the line $\mathrm{X}$ does not show visible changes, but, large values are obvious along the positive direction when moving the vehicle from an idling state and along the negative direction during acceleration. Also, in line $\mathrm{Y}$, large values are obvious along the positive direction when turning right, and along the negative direction when turning left. Likewise, by detecting the significant changes in the acceleration data, we can distinguish the running states from the image sensor.

Fig. 13 shows readings from the acceleration sensor starting from the idling state, turning right, moving forward for a while, and then turning left.

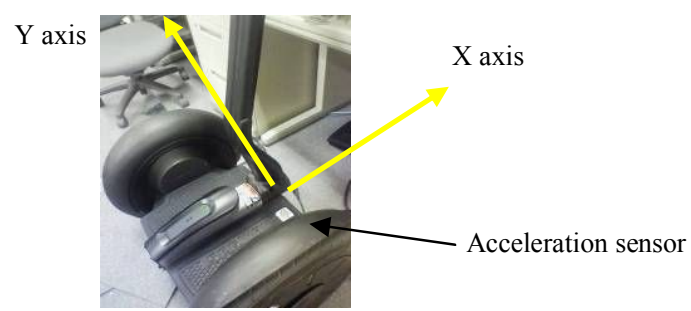

Fig. 12. Three axial directions

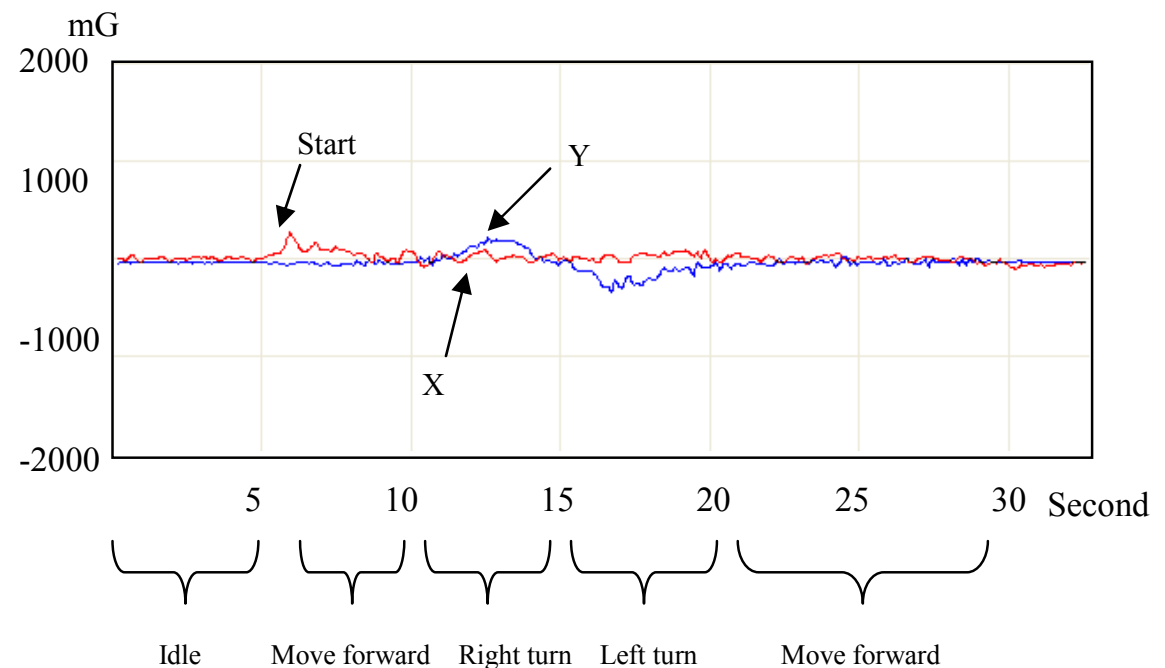

Fig. 13. An example of an acceleration sensors data

\subsection{Integrating two sensors information}

This section compares image sensor data and acceleration sensor data obtained simultaneously while riding the Segway. Fig. 14 shows the readings while the 
Segway is moving forward. When no moving objects are in the sight, the image sensor simply detects the optical flow for "moving forward" as explained in section 4.1. No remarkable changes can be verified from the acceleration sensor data. In contrast, Fig. 15 shows the case when the moving object exists in front of the Segway. In this case, the optical flow shows different trends from that in Fig. 14, although the acceleration sensor obtains similar data with Fig. 14. The moving object which affects the optical flows can be detected by examining the moment when such discrepancy occurs.

Fig. 16 and 17 show examples while the vehicle is turning right. In this case, acceleration sensor data shows the same trend, although optical flow shows different trends.
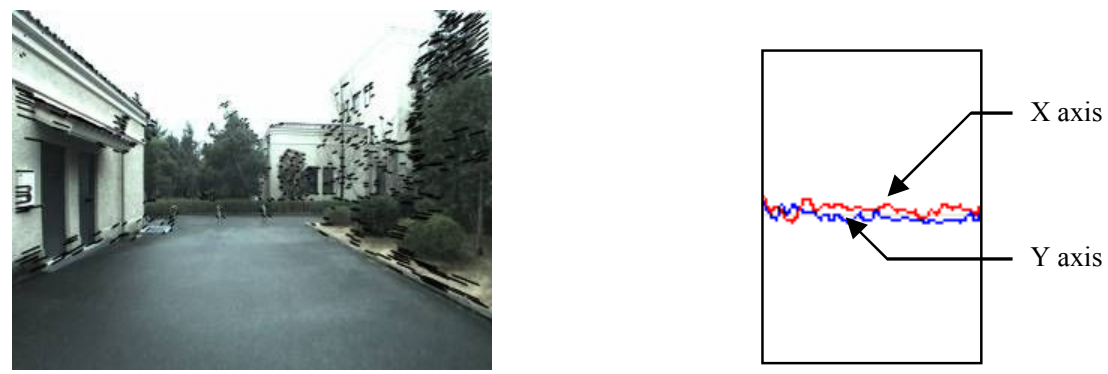

Fig. 14. Two sensors data when a moving object does not appear (moving forward)
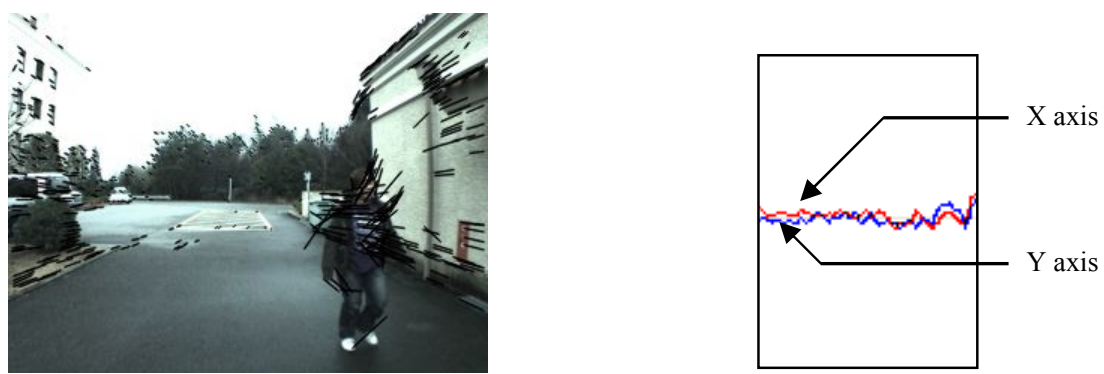

Fig. 15. Two sensors data when a moving object appears (moving forward)
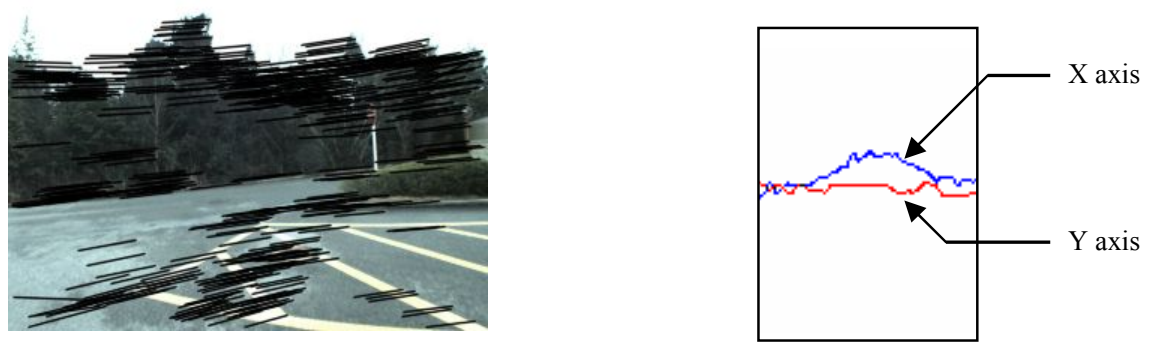

Fig. 16. Two sensors data when a moving object does not reflect (turning right) 

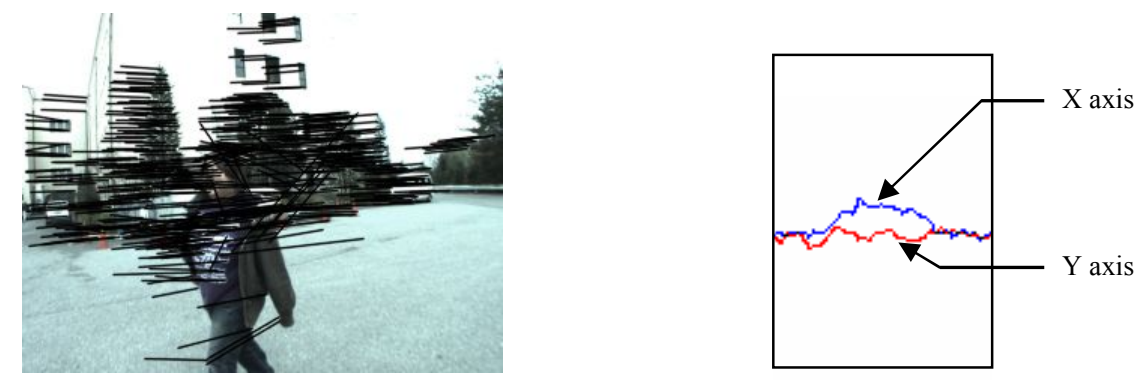

Fig. 17. Two sensors data when a moving object does not reflect (turning right)

\section{Conclusion}

This paper described our method to detect front moving objects by using the incompatibility between readings from an image sensor and an acceleration sensor. Moreover, the detection method of running state using two sensors to detect a moving object is described. As future work, it is necessary to examine whether the safety of the personal vehicle can be achieved with our method.

\section{Reference}

1. Segway, http://www.segway.com

2. B.K.P Horn and B.G Schunck, "Determining Optical Flow", Artifical Intelligence, Vol.17, pp.185-204, 1981

3. J.Y.Bouguet, "Pyramidal Implementation of the Lucas Kanade Feature Tracker", Intel Corporation, Microprocessor Research Labs, 2000

4. K. Onoguchi, "Shadow Elimination Method for Moving Object Detection," Proceedings of International Conference on Pattern Recognition (ICPR'98), pp.583-587, 1998

5. R.Okada, et al, "Obstacle Detection using Progressive Invariant and Vanishing Lines”, ICCV, pp.330-337, 2003

6. Point Grey Research, http://www.ptgrey.com/products/grasshopper/index.asp

7. ATR-Promotions, http://www.atr-p.com/sensor01.html 